\title{
The continuum from extreme conditions to the intensive care unit
}

\author{
Claude Guérin ${ }^{1,2}$
}

Affiliations: 'Service de Réanimation Médicale, Hôpital de la Croix-Rousse, Hospices Civils de Lyon, Lyon, France. ${ }^{2}$ Faculté de médecine Lyon Est, Université de Lyon, Lyon, France.

Correspondence: Claude Guérin, Service de Réanimation Médicale, Hôpital de la Croix-Rousse, Hospices Civils de Lyon, 103 Grande rue de la Croix Rousse, 69004 Lyon, France. E-mail: claude.guerindachu-lyon.fr

○ @ERSpublications

The ICU is a similar extreme environment to those people may put themselves in within the normal environment http://ow.ly/BDTJa

Just by changing their lifestyle, humans are challenging the respiratory system to an extent never previously reached; by exposing it to extreme conditions. In an article in this issue of the European Respiratory Review, ADIR and BOVE [1] have deciphered the conditions that may promote lung injury when the respiratory system is exposed to low atmospheric pressure, high pressure surrounding the body and extreme exercise. The intensive care unit (ICU) can also be seen as an extreme environment for the respiratory system. This is because ICU management is required for the most severe forms of acute lung disease, in particular acute respiratory distress syndrome (ARDS), and the components of the management may be extreme, such as prone positioning [2] or extracorporeal lung oxygenation [3]. Therefore, I would like to discuss some aspects of hypoxaemia and mechanical ventilation that occur in the ICU, and which closely link to the cases described by ADIR and BOVE [1].

The main advance in ARDS management is the limitation of pressure and volume [4], which is delivered by the ventilator and applied to the respiratory system, with the primary goal of minimising ventilator-induced lung injury (VILI) [5]. Using a lower tidal volume (targeting $6 \mathrm{~mL} \cdot \mathrm{kg}^{-1}$ predicted body weight), set by the ventilator, has been shown to improve survival to a two-fold higher volume in higher compared to lower tidal volume [4]. Targeting modest oxygenation (arterial oxygen tension $\left(\mathrm{PaO}_{2}\right) 55-80 \mathrm{mmHg}$ ) is also a component of lung-protective mechanical ventilation. The rationale for this is four-fold. First, targeting higher $\mathrm{PaO}_{2}$ (e.g. $100 \mathrm{mmHg}$ ) would commit the clinician to use potential harmful values of ventilator settings. For instance, higher positive end-expiratory pressure (PEEP) may worsen haemodynamics. A meta-analysis of three large randomised controlled trials comparing higher with lower PEEP found that the former was associated with higher survival in ARDS, but with a trend towards lower survival in patients with acute lung injury, i.e. patients with less severe hypoxaemia [6]. Secondly, avoiding hypoxaemia does not imply prevention of VILI. In fact, the opposite may even be true. Indeed, in the ARMA trial [4], the group of patients with acute lung injury and ARDS receiving lower tidal volume had worse oxygenation during the first week but eventually had a better outcome than the group with a higher tidal volume. Thirdly, hypoxaemia is not a primary cause of death in ARDS [7]. Finally, it has been claimed that very profound hypoxaemia may be well tolerated by referring to a seminal study performed in four mountaineers who climbed Mount Everest and in whom, for the first time in history, a direct measurement of arterial blood gas was performed while there [8]. $\mathrm{PaO}_{2}$ and arterial oxygen saturation were in the ranges of 19.1-29.5 mmHg and 34.4-69.7\%, respectively. However, it is difficult to translate these data to ARDS patients because in the mountaineers, hypoxaemia was not acute and oxygen transport was maintained as a

Received: Sept 032014 | Accepted: Sept 152014

Conflict of interest: None declared.

Provenance: Submitted article, peer reviewed.

Copyright OERS 2014. ERR articles are open access and distributed under the terms of the Creative Commons Attribution Non-Commercial Licence 4.0. 
result of the increase in haemoglobin concentration due to acclimatisation to high altitude over several weeks. One would argue that ARDS patients with severe hypoxaemia may benefit from blood transfusion to increase oxygen transport. A randomised controlled trial in ICU patients with anaemia compared profuse use of blood transfusion (target haemoglobin level $90 \mathrm{~g} \cdot \mathrm{L}^{-1}$ ) versus conservative use (haemoglobin level $70 \mathrm{~g} \cdot \mathrm{L}^{-1}$ ) and found that the first strategy was harmful in terms of organ dysfunction [9]. Therefore, the current policy in the ICU is to reduce the use of blood transfusion. However, a large number of ICU patients still receive blood transfusions. Furthermore, to date, transfusion-related acute lung injury may be a main cause of ARDS [10]. Finally, trials aiming at maximising oxygen transport in ICU patients did not find any benefits and were even harmful $[11,12]$. A word of caution to the contention that low levels of hypoxaemia are safe has been pointed out with the re-analysis of a large randomised controlled trial on fluid resuscitation in ARDS patients [13]. It has been shown that even mild hypoxaemia can be associated with long-term cognitive dysfunction in ARDS patients [13]. In fact, it should be noted that there is no prospective randomised controlled study comparing different oxygenation targets on patient outcome in ARDS.

ADIR and BOvE [1] mention the stress capillary failure, initially described by WeST et al. [14], as breaks in the alveolar-to-capillary membrane due to high hydrostatic lung microvascular pressure resulting from high cardiac output, which is achieved during intense exercise. Stress capillary failure has many implications in the ICU. First in the pathogenesis of high-altitude pulmonary oedema and neurogenic oedema, as underlined by ADIR and BOVE [1], and secondly in the pathophysiology of VILI. DREYFUSS and SAUMON [15] found similar alveolar-to-capillary membrane disruptions in rats with previously normal lungs who were receiving a large tidal volume during positive pressure mechanical ventilation. Therefore, VILI and stress capillary failure share a similar structural background. Furthermore, DREYFUSS and SAUMON [15] found that the main determinant of VILI was the total end-inspiratory lung volume and not the peak airway pressure. The relevant pressure that relates to change in lung volume is the transpulmonary pressure (Ptp), i.e. the

a)

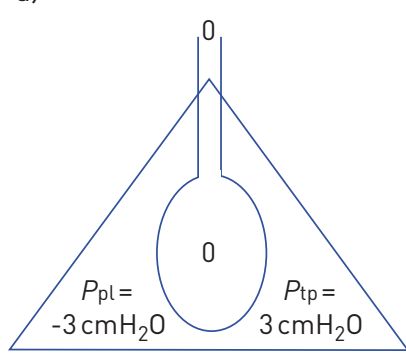

d)

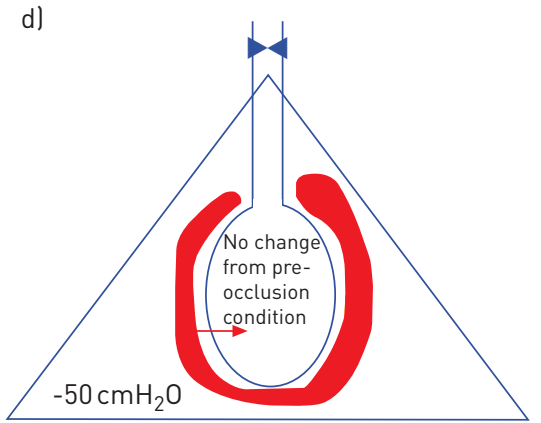

b)

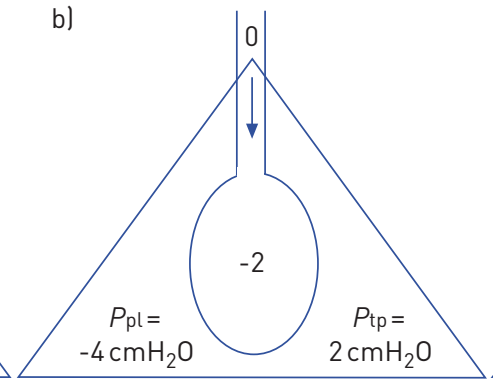

c)

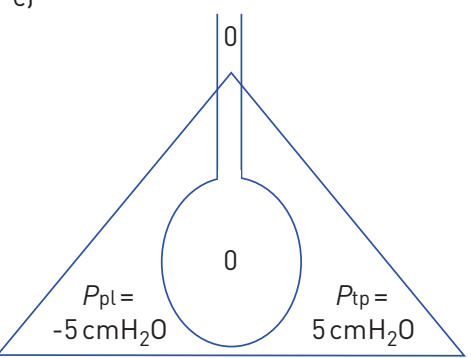

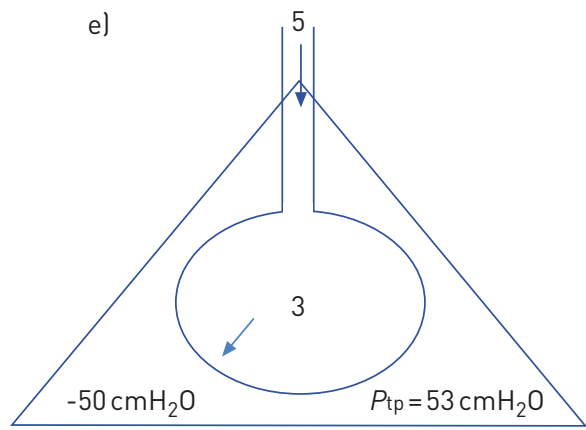

FIGURE 1 a) Breath-holding end-expiration: pleural $(P \mathrm{pl})$ and transpulmonary $\left(P_{\mathrm{tp}}\right)$ pressures in normal subject at the end of expiration. When the glottis is open and there is no flow at the mouth (breath-holding) the alveolar pressure is in equilibrium with atmospheric pressure. b) Inspiration: $P$ pl and $P$ tp during inspiration in a normal subject. $P_{\mathrm{pl}}$ becomes more negative with the inspiratory efforts. Alveolar pressure becomes lower than atmospheric pressure allowing air to flow from the atmosphere to the alveoli. c) Breath-holding at end-inspiration: $P_{\mathrm{pl}}$ and $P$ tp at the end of inspiration with breath-hold. Lung volume has increased by accommodating the tidal volume and Ptp is greater than at the end of expiration. d) Inspiration: complete and fixed upper airway obstruction. There is no communication between the atmosphere and the alveoli. The changes in $P$ pl are transmitted to the alveoli but the lung volume does not change. The hydrostatic microvascular pressure gradient between lung capillaries (red) and alveoli increases promoting an increase in fluid filtration across the alveolar-to-capillary membrane (arrow). e) End-inspiration: $P$ pl and $P$ tp in a patient receiving a low level of pressure support ventilation. The high $P$ tp values that would result from this condition may not be recognised as $P$ pl is not routinely measured in the intensive care unit. The key variable to monitor in such situations, in the perspective of ventilator-induced lung injury prevention, is the expired tidal volume that must be maintained at $\sim 6 \mathrm{~mL} \cdot \mathrm{kg}^{-1}$ predicted body weight. Numbers within the lung refer to alveolar pressure, and those at the proximal tube refer to atmospheric pressue. 

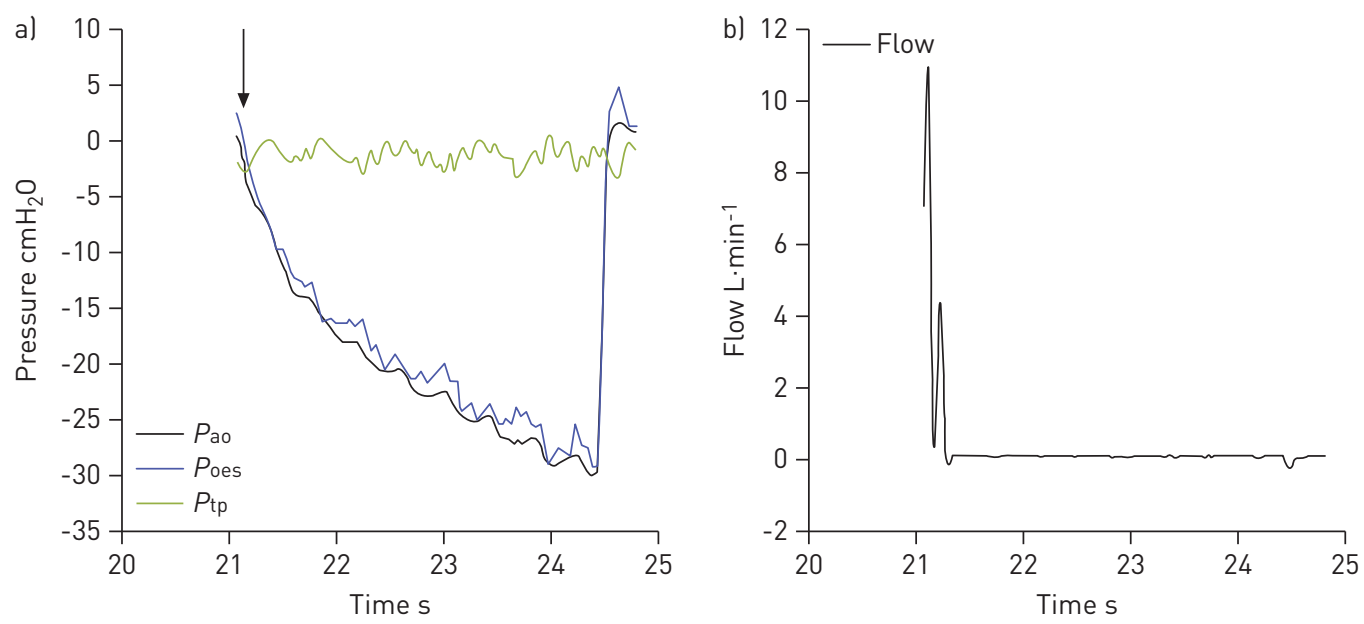

FIGURE 2 Occlusion test to ascertain the correct position of the oesophageal balloon in the thorax of a pig. a) Trace of airway pressure $\left(P_{\mathrm{aoo}}\right)$, oesophageal pressure $\left(P_{\mathrm{oes}}\right)$ and transpulmonary pressure $\left(P_{\mathrm{tp}}\right)$, and $\left.\mathrm{b}\right)$ flow in a pig that has been tracheotomised and is being mechanically ventilated. At the time indicated (a, arrow), the airways were occluded and the animal developed very high atmospheric pressure, during which time there was no change in flow and, therefore, in lung volume. The change in Poes parallels that in Pao, validating the good position of the oesophageal device. It can be seen that $P$ tp does not change (unpublished observation).

difference between alveolar and pleural pressure (fig. 1a-c). Thirdly, as mentioned previously, the prevention of VILI is the main goal for any caregiver setting the ventilator in ARDS patients. In subjects with normal lungs who are receiving mechanical ventilation for elective surgery [16] or in patients without ARDS mechanically ventilated in the ICU [17], the use of a lower tidal volume and setting PEEP at $5 \mathrm{cmH}_{2} \mathrm{O}$ was also found to be beneficial. Thus, there are a large number of conditions that require lungprotective mechanical ventilation. Finally, the limit between cardiogenic and non-cardiogenic pulmonary oedema is not clear as high microvascular pressure can induce ARDS. This feature has been taken into account in the recent definition of ARDS [18].

ADIR and BOVE [1] discuss negative pressure pulmonary oedema (NPPE) in scuba diving and mention that it has also been reported in the field of anaesthesiology in patients with fixed and complete upper airway obstruction. Two mechanisms may explain NPPE and have been reviewed recently [19]. The first is the increase in central blood volume due to higher venous return promoted by negative pleural pressure swings, which, together with a concomitant decrease in alveolar pressure, increase the capillary-to-alveoli pressure gradient. This mechanism would lead to hydrostatic pulmonary oedema, as it favours filtration of fluid across an intact alveolar-to-capillary membrane towards the interstitial space overwhelming the lymphatic drainage, and then to the alveoli (fig. 1d). The second is that the stress capillary failure would lead to protein-rich pulmonary oedema. In NPPE, Ptp should not change and the same is true for lung volume, as the airway does not communicate with the atmosphere (fig. 2). The treatment of NPPE includes airway patency and positive pressure ventilation. However, strong inspiratory efforts through patent airways may also damage the lungs via another mechanism. In ICU patients undergoing pressure support ventilation at the time of mechanical ventilation weaning, $P$ tp can be very high when patient inspiratory efforts synchronise with mechanical breath delivery (fig. 1e). As a result, a large tidal volume may enter the lung with the risk of barotrauma. It is worth emphasising that high Ptp may occur even at low levels of pressure support set at the ventilator [20]. This high Ptp can remain unrecognised as $P$ tp is not routinely monitored in ICU patients. Therefore, attention must be paid to expired tidal volume, which is the key variable to monitor in patients under any pressure-regulated modes of mechanical ventilation.

In an extreme environment like the ICU, understanding the pathophysiological basis and management of acute lung diseases is of paramount importance. The same is true for conditions arising from extreme environments that we, as humans, choose to face.

\section{References}

Adir Y, Bove AA. Lung injury related to extreme environments. Eur Respir Rev 2014; 23: 416-426.

2 Guérin C, Reignier J, Richard JC. Prone positioning in the acute respiratory distress syndrome. N Engl J Med 2013; 369: 980-981.

3 Peek GJ, Mugford M, Tiruvoipati R, et al. Efficacy and economic assessment of conventional ventilatory support versus extracorporeal membrane oxygenation for severe adult respiratory failure (CESAR): a multicentre randomised controlled trial. Lancet 2009; 374: 1351-1363. 
Ventilation with lower tidal volumes as compared with traditional tidal volumes for acute lung injury and the acute respiratory distress syndrome. The Acute Respiratory Distress Syndrome Network. N Engl J Med 2000; 342: 1301-1308. Slutsky A, Ranieri M. Ventilator-induced lung injury. N Engl J Med 2013; 369: 2126-2136.

6 Briel M, Meade M, Mercat A, et al. Higher vs lower positive end-expiratory pressure in patients with acute lung injury and acute respiratory distress syndrome: systematic review and meta-analysis. JAMA 2010; 303: 865-873.

7 Montgomery AB, Stager MA, Carrico CJ, et al. Causes of mortality in patients with the adult respiratory distress syndrome. Am Rev Respir Dis 1985; 132: 485-489.

8 Grocott MP, Martin DS, Levett DZ, et al. Arterial blood gases and oxygen content in climbers on Mount Everest. N Engl J Med 2009; 360: 140-149.

9 Hebert PC. Transfusion requirements in critical care (TRICC): a multicentre, randomized, controlled clinical study. Transfusion Requirements in Critical Care Investigators and the Canadian Critical Care Trials Group. Br J Anaesth 1998; 81: Suppl. 1, 25-33

10 Vlaar AP, Juffermans NP. Transfusion-related acute lung injury: a clinical review. Lancet 2013; 382: $984-994$.

11 Gattinoni L, Brazzi L, Pelosi P, et al. A trial of goal-oriented hemodynamic therapy in critically ill patients. $N$ Engl J Med 1995; 333: 1025-1032.

12 Hayes MA, Timmins AC, Yau EH, et al. Elevation of systemic oxygen delivery in the treatment of critically ill patients. N Engl J Med 1994; 330: 1717-1722.

13 Mikkelsen ME, Christie JD, Lanken PN, et al. The adult respiratory distress syndrome cognitive outcomes study: long-term neuropsychological function in survivors of acute lung injury. Am J Respir Crit Care Med 2012; 185: 1307-1315.

14 West JB, Mathieu-Costello O. Stress failure of pulmonary capillaries: role in lung and heart disease. Lancet 1992; 340: 762-767.

15 Dreyfuss D, Saumon G. Ventilator-induced lung injury: lessons from experimental studies. Am J Respir Crit Care Med 1998; 157: 294-323.

16 Futier E, Pereira B, Jaber S. Intraoperative low-tidal-volume ventilation. N Engl J Med 2013; 369 : 1862-1863.

17 Serpa Neto A, Nagtzaam L, Schultz MJ. Ventilation with lower tidal volumes for critically ill patients without the acute respiratory distress syndrome: a systematic translational review and meta-analysis. Curr Opin Crit Care 2014; 20: $25-32$.

18 Ranieri VM, Rubenfeld GD, Thompson BT, et al. Acute respiratory distress syndrome. The Berlin Definition. JAMA 2012; 307: E1-E8.

19 Lemyze M, Mallat J. Understanding negative pressure pulmonary edema. Intensive Care Med 2014; 40: 1140-1143.

20 Leray V, Bourdin G, Flandreau G, et al. A case of pneumomediastinum in a patient with acute respiratory distress syndrome on pressure support ventilation. Respir Care 2010; 55: 770-773. 